\title{
The influence of cement thickness on stem subsidence and cement creep in a collarless polished tapered stem WHEN ARE THICK CEMENT MANTLES DETRIMENTAL?
}

\section{E. Takahashi, \\ A. Kaneuji, \\ R. Tsuda, \\ Y. Numata, \\ T. Ichiseki, \\ K. Fukui, \\ N. Kawahara}

Kanazawa Medical

University, Kahoku-gun, Ishikawa, Japan

- E. Takahashi, MD, PhD,

Assistant Professor,

A. Kaneuji, MD, PhD, Professor,

- R. Tsuda, MD, PhD,

Assistant Professor,

Y. Numata, MD, Clinical Fellow,

- T. Ichiseki, MD, PhD,

Associate Professor,

- K. Fukui, MD, PhD,

Senior Assistant Professor,

- N. Kawahara, MD, PhD,

Professor, Department of

Orthopaedic Surgery, Kanazawa

Medical University, Daigaku,

Uchinada-machi, Kahoku-gun,

Ishikawa 920-0293, Japan.

Correspondence should be sent to

Dr A. Kaneuji; e-mail:

kaneuji@kanazawa-med.ac.jp

doi: $10.1302 / 2046-3758.65 . B J R-$ 2017-0028.R1

Bone Joint Res 2017;6:351-357.

Received: 27 January 2017;

Accepted: 14 March 2017

\section{Objectives}

Favourable results for collarless polished tapered stems have been reported, and cement creep due to taper slip may be a contributing factor. However, the ideal cement thickness around polished stems remains unknown. We investigated the influence of cement thickness on stem subsidence and cement creep.

\section{Methods}

We cemented six collarless polished tapered (CPT) stems (two stems each of small, medium and large sizes) into composite femurs that had been reamed with a large CPT rasp to achieve various thicknesses of the cement mantle. Two or three tantalum balls were implanted in the proximal cement in each femur. A cyclic loading test was then performed for each stem. The migration of the balls was measured three-dimensionally, using a micro-computed tomography (CT) scanner, before and after loading. A digital displacement gauge was positioned at the stem shoulder, and stem subsidence was measured continuously by the gauge. Final stem subsidence was measured at the balls at the end of each stem.

\section{Results}

A strong positive correlation was observed between mean cement thickness and stem subsidence in the CT slices on the balls. In the small stems, the balls moved downward to almost the same extent as the stem. There was a significant negative correlation between cement thickness and the horizontal:downward ratio of ball movement.

\section{Conclusion}

Collarless polished tapered stems with thicker cement mantles resulted in greater subsidence of both stem and cement. This suggests that excessive thickness of the cement mantle may interfere with effective radial cement creep.

Cite this article: Bone Joint Res 2017;6:351-357.

Keywords: Total hip arthroplasty, Polished tapered stem, Cement thickness

\section{Article focus}

- Reliable information is currently unavailable on whether cement thickness affects radial cement creep for cemented polished tapered stems.

- Our study was designed to investigate the influence of cement thickness on stem subsidence and cement creep using micro-CT.

\section{Key messages}

- In our biomechanical study, data from CT slices showed a strong positive correlation between subsidence of collarless polished tapered stems and mean cement thickness.
- There was a significant negative correlation between cement thickness and the horizontal:downward ratio of tantalum ball movement in the cement.

- Our findings suggest that excessively thick cement mantles are unlikely to provide ideal hoop stress or radial creep for stem subsidence.

\section{Strengths and limitations}

Strengths: We reproduced the environment within the human body in terms of moistness and temperature, and we included unloading "sleep" periods to 
provide stress relaxation for the cement. Precise micro-CT was used for measurements.

- Limitations: Cement movement in this study was limited to the tracking of tantalum ball migration within the cement, rather than the movement of the cement itself.

\section{Introduction}

Since the cement fixation system of femoral stems for total hip arthroplasty (THA) was established in the 1960s, a variety of different concepts have been applied to the development of the associated implants. Recent studies of cemented stems have reported different outcomes depending on the surface finish. ${ }^{1,2}$ Bedard et $\mathrm{al}^{3}{ }^{3}$ in $\mathrm{a}$ review of long-term clinical outcomes over more than 20 years suggested superior outcomes for polished surface stems. According to Lee et al, ${ }^{4}$ the polished tapered stems slipped in the cement and created considerable compressive stress at the bone-cement interface, but with little shear stress. Kaneuji et $\mathrm{al}^{5}$ verified this theory using a biomechanical model, and demonstrated that the polished tapered stems were associated with greater subsidence and compressive force at the bone-cement interface than was seen with rough surface stems. The authors also reported that thicker cement mantles led to greater subsidence of the stems and higher compressive force.

However, excessive subsidence of the stem has been associated with stem loosening, ${ }^{6}$ and it is still unclear whether thickening the cement mantle leads to better outcomes. Many studies have reported good outcomes for a cement mantle thickness of at least $2 \mathrm{~mm},{ }^{7-13}$ but a favourable outcome with a thin cement mantle was also reported for polished French stems. ${ }^{14,15}$ These findings are termed the "French paradox", and the reason for the outcome remains unclear. Up to this point, no basic experiments have been performed to investigate the appropriate thickness of the cement mantle for polished stems.

This study was conducted to investigate the subsidence of stem and cement, using polished tapered stems in varying thicknesses of cement mantle, and to measure three-dimensional (3D) cement creep in order to determine the optimal thickness of the cement mantle.

\section{Materials and Methods}

We used a biomechanical instrument that has already been reported by Kaneuji et $\mathrm{al}^{5}$ for this study, and measured stem subsidence after a loading test. We implanted tantalum balls in the cement and measured their movement with a micro-CT scanner. Details of the methodology are described below.

Prosthesis. We used clinically available collarless polished tapered (CPT) stems (Zimmer Biomet, Warsaw, Indiana) with less than $0.1 \mu \mathrm{m}$ in surface roughness. The

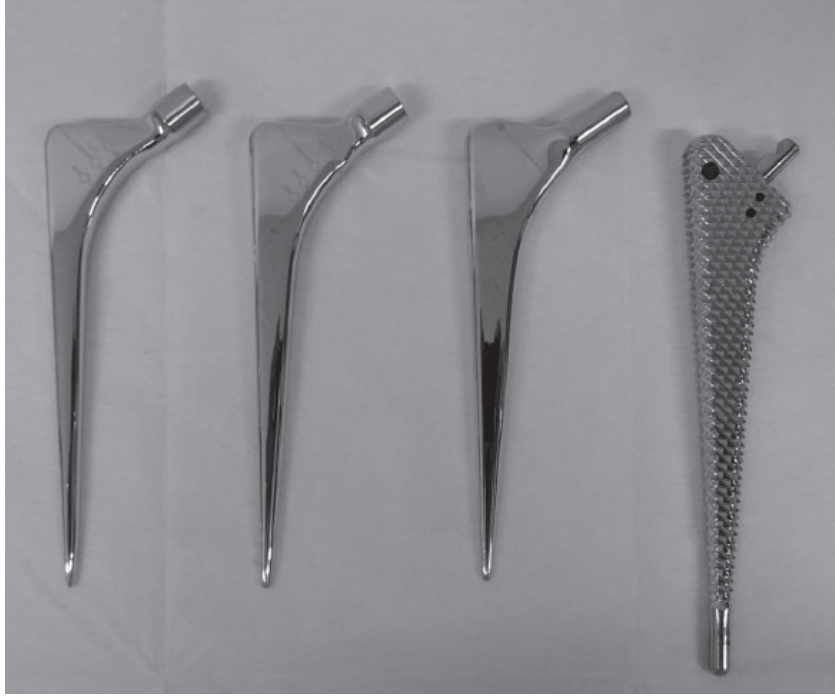

Fig. 1

CPT stems and No. 3 rasp. These are CPT stem sizes small, medium and large, respectively, from the left (right, the No. 3 large rasp).

stems were tapered from proximal to distal on both the frontal and lateral sides. This study used a total of six CPT stems: two each of small, medium and large (Fig. 1). The most proximal transverse diameter increased by $2.5 \mathrm{~mm}$, and the offset diameter increased by $2 \mathrm{~mm}$, between the small and medium stems and between the medium and large stems, respectively. The stems were made of cobalt-chromium ( $\mathrm{CoCr}$ ) alloy.

Composite femurs. We used composite femurs \#3403 (Pacific Research Laboratories, Inc., Vashon, Washington) that resembled human femurs in form and in mechanical and material characteristics. ${ }^{16}$ The composite femurs were cut at the femoral neck. All the femurs were reamed with a large No. 3 rasp (Zimmer Biomet), and CPT stems in different sizes were inserted to provide a variety of cement thicknesses. As a marker for 3D analysis by micro-CT, four tantalum balls $0.4 \mathrm{~mm}$ in diameter (Bal-tec, Micro Surface Engineering, Los Angeles, California) were embedded in the outer circumference of the composite femurs $1 \mathrm{~cm}$ proximal to just above the lesser trochanter (Fig. 2a and Fig. 2b). Similarly, tantalum balls $0.4 \mathrm{~mm}$ in diameter were embedded circumferentially near the tips of the stems (Fig. 2c) that were to be inserted into the femur models. Each ball-composite femur unit was then soaked with blended vegetable oil, made of rapeseed oil and soybean oil, (The Nissin Oillio group Ltd., Tokyo, Japan) for 24 hours (Fig. 2d) to mimic the wet conditions of the in vivo femoral environment.

Loading test equipment. The composite femurs were attached to the testing instrument, which was composed of S45C structural carbon steel and epoxy resin (Devcon B; ITW Industry Co. Ltd, Osaka, Japan). The epoxy resin was shaped in the same external form as the composite femurs. Bone cement (Osteobond; Zimmer Biomet) was 


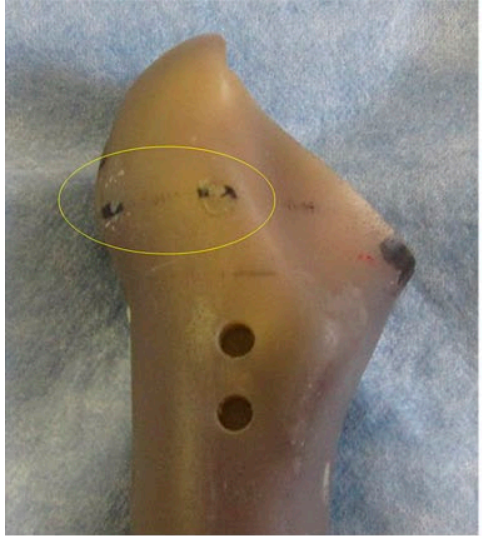

Fig. 2a

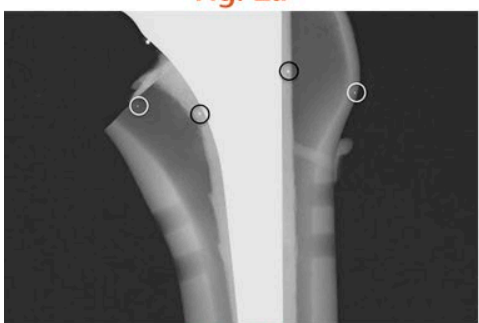

Fig. $2 \mathrm{~b}$

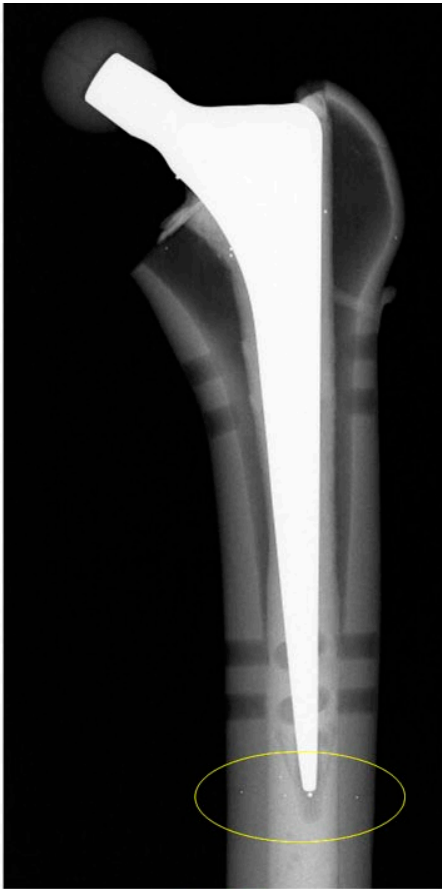

Fig. $2 c$

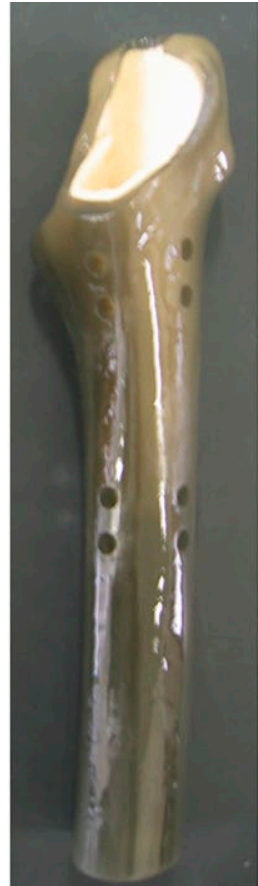

Fig. $2 d$

a) Figure showing small drilled holes for inserting tantalum balls; b) radiograph film after fixing stem with cement. Tantalum markers are visible on the outside of the composite femur (white circles) and in the cement (black circles); c) Radiograph showing placement of tantalum balls near the tips of the stems and d) Figure showing a ball-composite femur unit soaked in vegetable oil.

mixed in a vacuum cement mixing system, and $80 \mathrm{~g}$ of the mixed cement was inserted into the composite femur using a cement injection gun. Tantalum balls ( $1 \mathrm{~mm}$ in diameter) (Bal-tec, Micro Surface Engineering) were attached to the tips of the stems using glue (Fig. 2c). A centraliser was also attached before inserting the stem into the composite femur. A bone plug made of wax was fixed into the femoral canal. Before the cement solidified, two or three $0.6 \mathrm{~mm}$ tantalum balls were placed in the cement at approximately the same height as the markers embedded in the proximal position of the composite femur (Fig. 2b).

The hip prosthesis model was kept at $37^{\circ} \mathrm{C}$ to mimic the conditions of the in vivo environment. A temperature sensor (T-35, Takigen MFG Co., Ltd, Tokyo, Japan) was attached to the epoxy resin used to fix the composite femur, so that the temperature from a heater (G6A92 240V, 250W; Takigen) was maintained at $37^{\circ} \mathrm{C}$.

Methods and duration of loading. Loading was applied at an internal angle of $15^{\circ}$ on the coronal plane to the metal femoral head (Co-Cr, $26 \mathrm{~mm}$ in diameter), attached to the stem neck. ${ }^{17}$ Sine wave load (1 Hz, 3000 N) was applied 500000 times. This was estimated to be equivalent to the load of a six-month walk. ${ }^{18,19}$ The test load of $3000 \mathrm{~N}$ was considered to be equivalent to the load to the hip joint of a $70 \mathrm{~kg}$ person in a standing position on one leg. ${ }^{18}$ The loading tests were conducted using a fatigue strength testing system (EHF-UM 300KN-70L; Shimadzu
Corp., Kyoto, Japan). In this experiment, an eighthour unloading period was applied between the 16-hour loading periods to mimic an actual sleeping period in clinical settings. Unloading periods were considered to be important for stress relaxation of the cement.

Parameters and methods of testing: stem subsidence. Measurement data were obtained over time using a $5 \mathrm{~mm}$ digital displacement gauge (DTH-A5; Kyowa Electronic Instruments Co. Ltd, Tokyo, Japan) placed at the stem shoulder (Fig. 3). Data were sent automatically to a personal computer by means of data collection and analysis software (Sensor Interface PCD-300A; Kyowa). Each set of data, measurements collected for eight minutes every hour automatically, was sorted into a file to collect 10000 data sets, and the data were entered into the computer. A total of 216 files were extracted per loading test. The measurement data were adjusted and converted to units of distance. Each of the 16-hour loading and eight-hour unloading periods of each day were divided into the early, middle and late phases, and the first two files from each phase (a total of 20000 data sets) were used. Subsidence was defined as the mean of the maximum values of sine waves from the 20000 data sets of each phase of the loading period. The mean values of the 20000 data sets of the unloading period were also collected. Perpendicular migration of the tantalum balls at the end of the stems was measured by micro-CT, and this was used as the final amount of subsidence. 


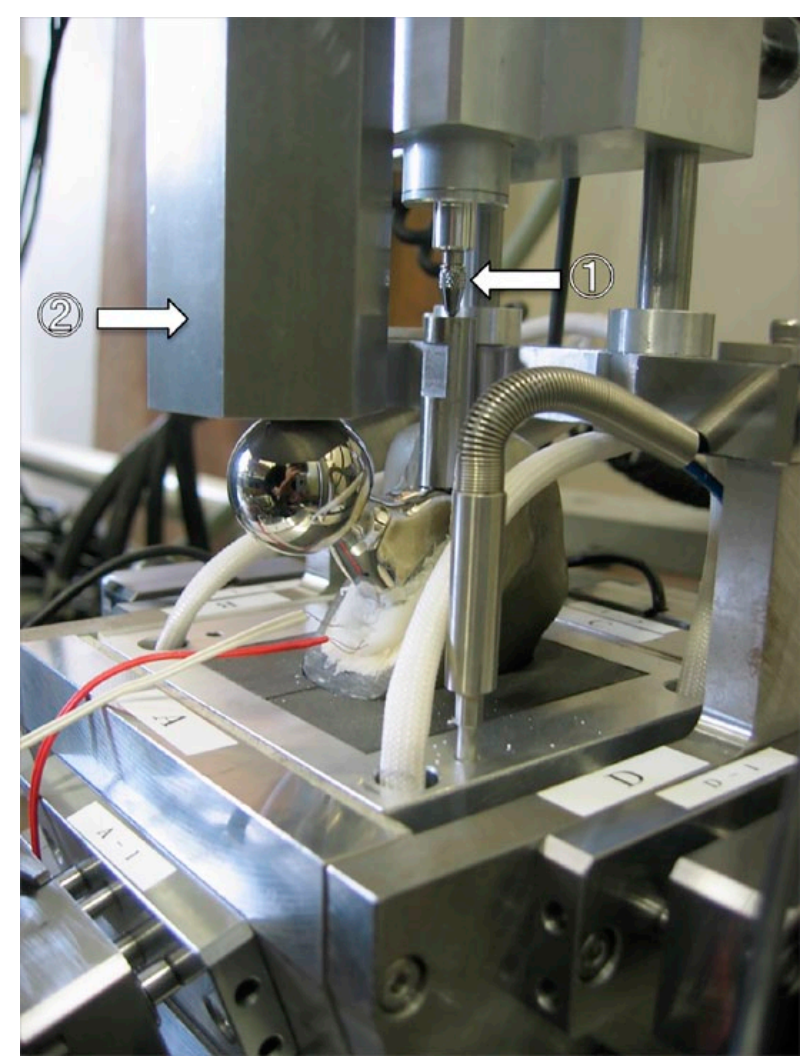

Fig. 3

The testing instrument. A composite femur with stem was inserted into the testing instrument. A metal head was attached to the stem neck and was placed in contact with a loading bar (2). A digital displacement gauge (1) was placed at the stem shoulder to measure stem motion continuously during loading and unloading periods.

Parameters and methods of testing: micro-CT analysis. Before and after loading, 3D images of the areas within a 2 $\mathrm{cm}$ radius of the markers were obtained using a micro-CT (Microfocus X-ray CT System) scanner (TOSCANER-32250 uhd; Toshiba IT \& Control Systems Corp., Tokyo, Japan) to measure the amount of migration for the tantalum balls within the cement and for the balls that were attached to the stem tip. Images were taken with a slice spacing of $95 \mu \mathrm{m}$. The maximum spatial resolution of the micro-CT scanner was $5 \mu \mathrm{m}$. In the cross-sectional CT images in which the tantalum balls were detected, the shortest distance of the line segment that intersected a ball between the bone and the stem was defined as "cement width." These images were also used to measure the shortest distance between the centre point of the anterior, posterior, medial and lateral surfaces of the stem and the canal bone. The mean value for these four measurements was defined as the "mean cement thickness" for each stem (Fig. 4).

The distance of the migration of a tantalum ball can represent the distance of cement movement in a specific location. With this measuring method, the embedded markers in the composite femur were used as a reference point. The position of a tantalum ball before applying

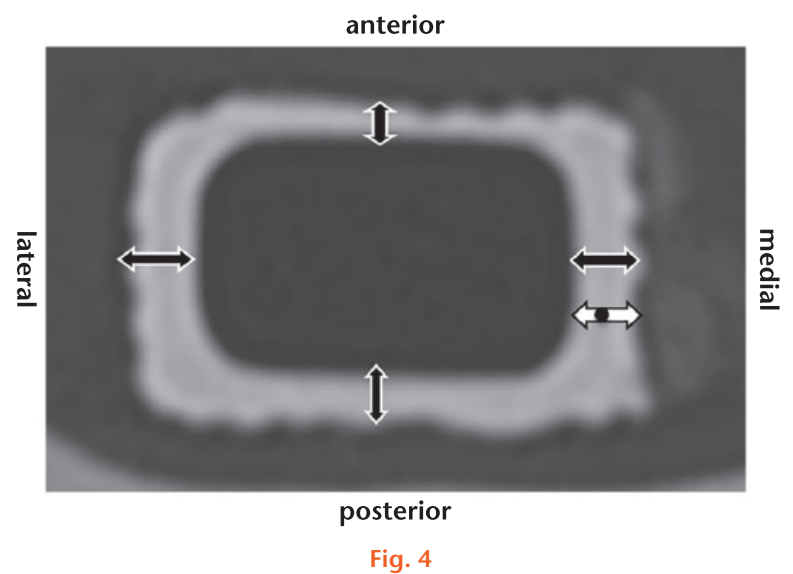

A micro-CT slice showing a tantalum ball as a black ball in the cement. The white arrow shows cement width. The mean value of the four black arrows was defined as "mean cement thickness".

load was defined as the original point, and the 3D coordinate values after applying load were calculated. The horizontal distance of the outward or inward migration related to the femoral medulla was expressed as "+" and "-", respectively. For the amount of perpendicular migration, data were obtained on the distance parallel to the axis of the bone. Movement in the direction of subsidence was expressed as "+".

Statistical analysis. The correlation between stem subsidence and mean cement thickness was investigated using Pearson's correlation coefficient. The relationship between the behaviour of tantalum balls and cement thickness was investigated using Spearman's rankcorrelation coefficient. A comparison of the movement of balls in two different thicknesses of cement was investigated using the Mann-Whitney $U$ test. A significance level of 0.05 was used for these tests. Statistical analysis was performed using StatView version 5.0 (SAS Institute Inc., Cary, North Carolina).

\section{Results}

Stem subsidence. A digital displacement gauge, placed at the stem shoulder during testing, showed that all stems subsided after cyclic loading (Fig. 5). This subsidence was partially reversed during the unloading periods, but increased gradually over time. The smaller stems tended to be associated with greater subsidence; each of the small stems subsided further than either of the large stems. The mean values and standard deviations (SD) for final subsidence, as measured at the balls attached to the tips of the stems, were $0.179 \mathrm{~mm}$ (SD 0.084) for the large stems, $0.375 \mathrm{~mm}$ (SD 0.091) for the medium stems, and $0.495 \mathrm{~mm}$ (SD 0.126) for the small stems.

Cement thickness. Mean cement thickness decreased as stem size increased: $1.52 \mathrm{~mm}$ (SD 0.45) for the large stems, $2.19 \mathrm{~mm}$ (SD 0.96) for the medium stems, and $2.57 \mathrm{~mm}$ (SD 1.61) for the small stems. A strong positive 


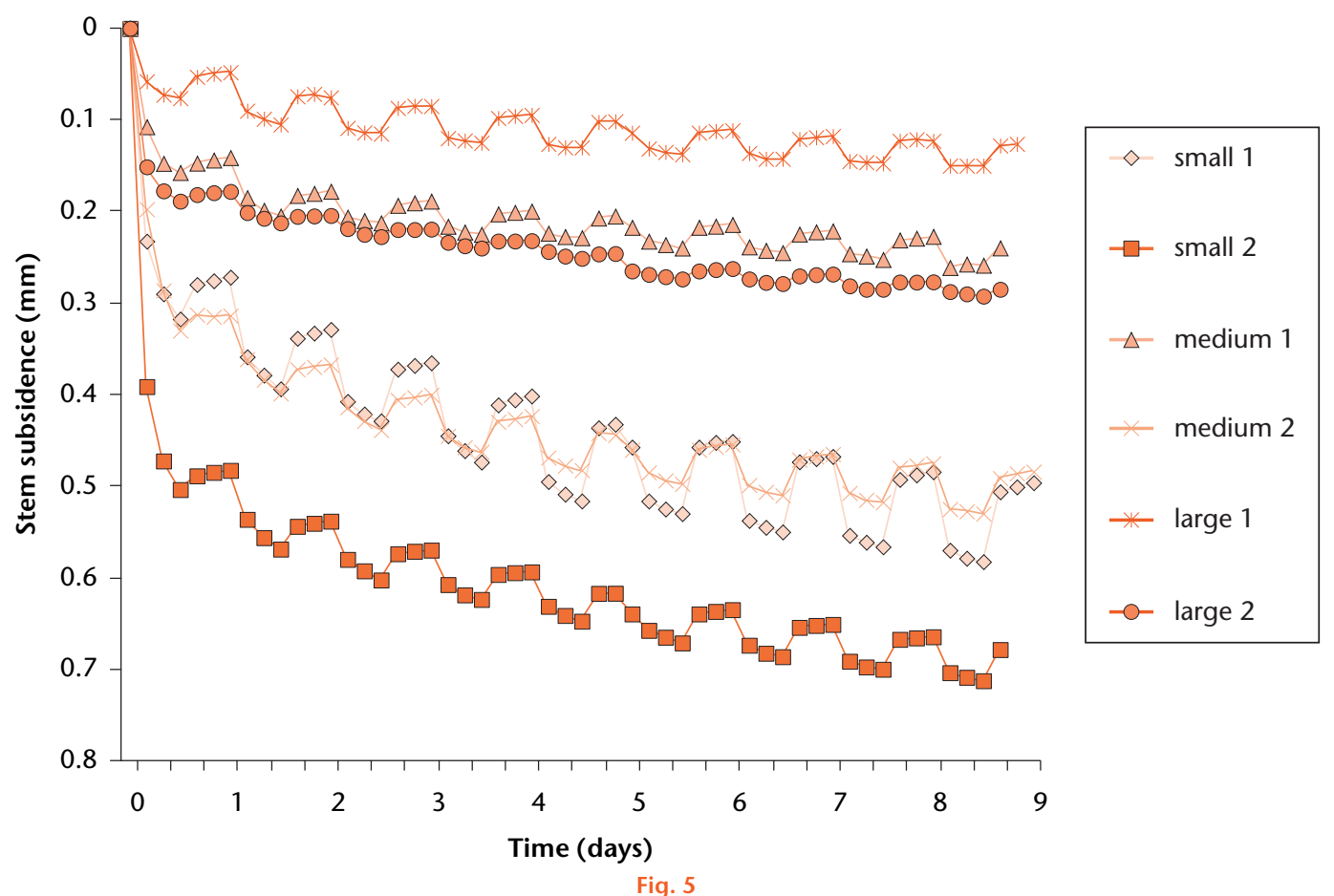

Graph showing stem subsidence. All stems subsided rapidly on the first day, after which subsidence continued gradually. All stems subsided down during loading periods and rose up during unloading periods. This stem motion was observed repeatedly on a daily basis during the testing of all stems.

correlation was observed between mean cement thickness and final stem subsidence measured by the ball at the end of stem, with less subsidence associated with a thinner cement mantle. (Fig. 6).

Investigation of the amount and direction of cement creep. A total of 13 tantalum balls (three for one of the two small stems, and two each for the other stems) were visualised in the micro-CT images. All of the visualised tantalum balls were found to have moved in the direction of stem subsidence. The mean distance of ball subsidence was $0.301 \mathrm{~mm}(0.015$ to 0.607 , sD 0.231). Table I shows measured and calculated values for different ball sizes.

The mean ratio of ball subsidence:stem subsidence was $102.9 \%$ (97.8\% to $113.5 \%$, SD $6.4 \%$ ) for small stems, $68.4 \%$ (19.6\% to $97.3 \%$, SD $36.7 \%$ ) for medium stems, and $38.2 \%$ (8.0\% to $92.4 \%$, SD $40.6 \%)$ for large stems. For small stems, the balls subsided downwards to almost the same extent as the stems.

The mean horizontal movement was $0.038 \mathrm{~mm}$ (-0.082 to 0.290, SD 0.103).

Eight tantalum balls moved outwards, and five moved inwards. Inward movement was noted in three of five tantalum balls for the small stems, two of four for the medium stems, and zero of four for the large stems. The mean horizontal:downward ratio of ball movement was $66.3 \%(-14.4 \%$ to $286.7 \%$, SD $102.2 \%)$ in all balls. This ratio for the large stems was $202 \%$ ( $146 \%$ to $287 \%$ ), indicating effective radial cement creep. The mean ratio for the small and medium stems was $-1.1 \%(-14.4 \%$ to

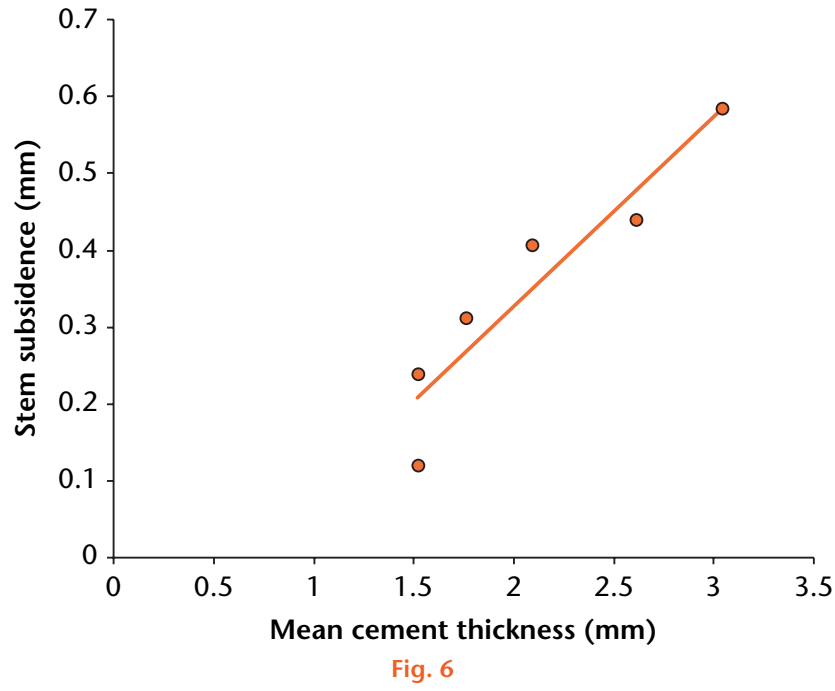

Graph showing the correlation between stem subsidence and cement thickness. This correlation diagram shows mean cement thickness and stem subsidence for each of six femurs. Pearson's correlation coefficient was $0.94(Y=0.2469 x-0.167, p \leq 0.05)$.

23.0\%), indicating that the thicker cement tended not to move in a horizontal direction by stem subsidence. Spearman's rank-correlation coefficient showed a negative correlation between the horizontal:downward ratio of ball movement and cement width (Spearman's rank correlation coefficient $\rho=-0.70, p<0.05$ ); thin cement mantle was associated with a higher percentage of outward movement. When compared to the movement of 
Table I. Measured and calculated values

\begin{tabular}{|c|c|c|c|c|c|c|c|c|c|c|c|c|c|}
\hline & \multicolumn{2}{|c|}{ Large 1} & \multicolumn{2}{|c|}{ Large 2} & \multicolumn{2}{|c|}{ Medium 1} & \multicolumn{2}{|c|}{ Medium 2} & \multicolumn{2}{|c|}{ Small 1} & \multicolumn{3}{|c|}{ Small 2} \\
\hline & Ball 1 & Ball 2 & Ball 1 & Ball 2 & Ball 1 & Ball 2 & Ball 1 & Ball 2 & Ball 1 & Ball 2 & Ball 1 & Ball 2 & Ball 3 \\
\hline $\begin{array}{l}\text { Horizontal movement } \\
\text { of ball }(\mathrm{A})(\mathrm{mm})\end{array}$ & 0.161 & 0.122 & 0.029 & 0.043 & 0.014 & -0.027 & -0.033 & 0.019 & -0.021 & 0.29 & -0.082 & 0.059 & -0.075 \\
\hline $\begin{array}{l}\text { Downward movement } \\
\text { of ball }(B)(\mathrm{mm})\end{array}$ & 0.11 & 0.055 & 0.019 & 0.015 & 0.061 & 0.189 & 0.427 & 0.422 & 0.461 & 0.41 & 0.571 & 0.607 & 0.575 \\
\hline Stem subsidence $(\mathrm{C})(\mathrm{mm})$ & \multicolumn{2}{|c|}{0.119} & \multicolumn{2}{|c|}{0.238} & \multicolumn{2}{|c|}{0.311} & \multicolumn{2}{|c|}{0.439} & \multicolumn{2}{|c|}{0.406} & \multicolumn{3}{|c|}{0.584} \\
\hline $\mathrm{A} / \mathrm{B}(\%)$ & 146.4 & 221.8 & 152.6 & 286.7 & 23.0 & -14.3 & -7.7 & 4.5 & -4.6 & 70.7 & -14.4 & 9.7 & -13.0 \\
\hline $\mathrm{B} / \mathrm{C}(\%)$ & 92.4 & 46.2 & 8.0 & 6.3 & 19.6 & 60.8 & 97.3 & 96.1 & 113.5 & 101.0 & 97.8 & 103.9 & 98.5 \\
\hline Cement width (mm) & 2.28 & 1.33 & 1.02 & 1.52 & 1.9 & 2.66 & 3.99 & 2.28 & 1.52 & 1.52 & 5.32 & 4.18 & 1.9 \\
\hline Mean cement thickness (mm) & 1.52 & & 1.52 & & 1.76 & & 2.61 & & 2.09 & & 3.04 & & \\
\hline
\end{tabular}

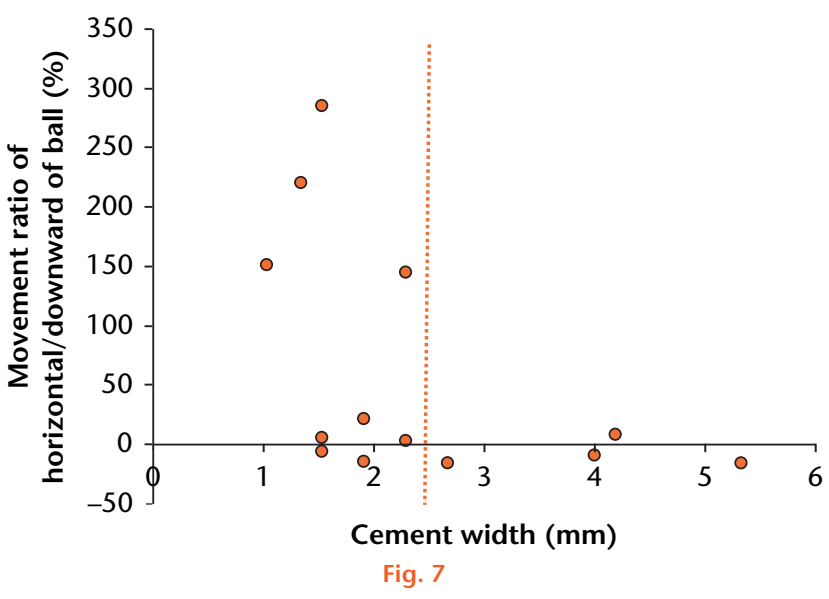

A diagram of the horizontal:downward ratio of ball movement and cement width. This diagram demonstrated that effective horizontal cement movement in relation to cement subsidence was seen for cement widths of less than $2.5 \mathrm{~mm}$ (dotted line).

balls in two different thicknesses of cement $(\geq 2.5 \mathrm{~mm}$ and $<2.5 \mathrm{~mm}$ ), we found a significant difference in the horizontal:outward ratio of movement between the two groups ( $p=0.03$ ); outward movement of the balls was observed significantly more frequently for the cement width of $<2.5 \mathrm{~mm}$ (Fig. 7).

\section{Discussion}

Currently, at least $2 \mathrm{~mm}$ of cement width is recommended for cemented stem fixation, and this recommendation is widely accepted. ${ }^{7-13}$ A thin cement mantle is considered to be less capable of absorbing energy and to be associated with poor outcomes, with potential for cracking and breakage. ${ }^{20}$ Localised cement defects have reportedly been associated with osteolysis and/or stem loosening, because the defect allows a passage for wear debris particles to travel from the stem-cement interface to the cement-bone interface. ${ }^{21}$

These findings support the recommendation for a cement mantle thickness of $\geq 2 \mathrm{~mm}$. However, many reports have been based on rough-surface stems, and it has been unclear whether the same findings would be observed for polished-surface stems. El Masri et al ${ }^{15}$ reported that polished collared tapered stems, inserted using a line-to-line cementing technique to create a thin mantle, were associated with favourable long-term results. This phenomenon is called the "French paradox". However, increased loosening has been reported for French rough-surface stems. ${ }^{14}$ This implies that it may be important for rough-surface stems to be used with a cement layer at least $2 \mathrm{~mm}$ in thickness.

To date, very few studies have reported on the question of whether the cement thickness for polished tapered stems should indeed be $\geq 2 \mathrm{~mm}$. Scheerlinck et $\mathrm{al}^{22}$ conducted CT analysis of femoral hip implants that had been inserted line-to-line versus one-size undersized in paired cadaver femurs, and found that the lineto-line stems resulted in better alignment. Moreover, the mean thickness of the cement mantle for the stems inserted in a line-to-line fashion was $3.08 \mathrm{~mm}$, while cement defects defined as $\leq 1 \mathrm{~mm}$ in thickness were found in only $6.2 \%$ of the cement. ${ }^{22}$ This suggests that actual cement defects may not be significant even if cement layers appear thin in radiographs. Janssen et al 23 found that, based on finite element analysis, stems inserted in a line-to-line fashion were associated with more favourable rotation stability and less cement damage than the one-size undersized stems.

In polished stems, Lee et $\mathrm{al}^{4}$ reported that a strong compressive force was exerted at the bone-cement interface as the polished stem slipped within the cement. The present study on collarless polished tapered stems supports the theory that effective radial cement creep (i.e. outward movement of balls) is more likely to occur if the cement mantle thickness is $\leq 2.5 \mathrm{~mm}$.

Our findings also suggest that, if the cement mantle were too thick no effective radial compression would occur and the cement would subside along with the stem. This leads to the conclusion that undersized cemented stems are not desirable. Physically, cement is considered to be strong in compression but weak in shear and tensile stress.4,24-26 The use of undersized polished tapered stems could therefore be associated with cement breakage or stem loosening because shear stress is exerted on the cement as the stem subsides.

Stem subsidence and cement creep may be affected by the different surface finish of stems. A recent basic experiment of similar composite femurs showed that a cement mantle thickness of $\geq 5 \mathrm{~mm}$ could lead to 
cracking at the cement-bone interface for Charnley and Lubinus SP II stems. ${ }^{27}$ This suggests that the use of undersized stems for the purpose of attaining greater cement thickness could also increase the risk of stem failure in the non-polished stems.

The present study has the following potential limitations: this is a biomechanical study, not a human body; load was applied in one direction only. Actual hip motion is not in a single constant direction; observations were limited to the tracking of tantalum ball migration in the cement rather than the actual movement of the cement itself; different alignment of the inserted stems may yield different results; no loosening of the stems was observed after testing, which could mean no difference in durability based on cement thickness; and this study used CPT stems; the use of other types of stem or cement might not yield the same results. In addition, the study did not address the behaviour of stems and cement in instances of cement defects or a very thin cement mantle.

Nevertheless, the study results may provide important basic data for polished tapered stems. Our findings suggest that excessively thick cement mantles due to the use of undersized stems are unlikely to provide ideal hoop stress or radial creep associated with subsidence of the stems.

\section{References}

1. Collis DK, Mohler CG. Comparison of clinical outcomes in total hip arthroplasty using rough and polished cemented stems with essentially the same geometry. J Bone Joint Surg [Am]2002;84-A:586-592.

2. Meding JB, Nassif JM, Ritter MA. Long-term survival of the T-28 versus the TR-28 cemented total hip arthroplasties. J Arthroplasty 2000;15:928-933.

3. Bedard NA, Callaghan JJ, Stefl MD, Liu SS. Systematic review of literature of cemented femoral components: what is the durability at minimum 20 years followup? Clin Orthop Relat Res 2015;473:563-571.

4. Lee AJC, Parkins RD, Ling RSM. Time-dependent properties of polymethylmethacrylate bone cement: the interaction of shape of femoral stems, surface finish and bone cement. In: Older J, ed. Implant bone interface. London: Springer-Verlag, 1990:85-90.

5. Kaneuji A, Yamada K, Hirosaki K, Takano M, Matsumoto T. Stem subsidence of polished and rough double taper stems: in vitro mechanical effects on the cementbone interface. Acta Orthop 2009;80:270-276.

6. Karrholm J, Borssen B, Lowenhielm G, Snorrason F. Does early micromotion of femoral stem prostheses matter?: 4-7-year stereoradiographic follow-up of 84 cemented prostheses. J Bone Joint Surg [Br] 1994;76-B:912-917.

7. Ebramzadeh E, Sarmiento A, McKellop HA, Llinas A, Gogan W. The cement mantle in total hip arthroplasty. Analysis of long-term radiographic result. J Bone Joint Surg [Am]1994;76-A:77-87.

8. Jasty M, Maloney WJ, Bragdon CR, et al. The initiation of failure in cemented femoral components of hip arthroplasties. J Bone Joint Surg [Br] 1991;73-B:551-558

9. Mann KA, Gupta S, Race A, et al. Cement microcracks in thin-mantle regions after in vitro fatigue loading. J Arthroplasty 2004;19:605-612.

10. Mulroy WF, Estok DM, Harris WH. Total hip arthroplasty with use of so-called second-generation cementing techniques. A fifteen-year-average follow-up study. J Bone Joint Surg [Am]1995;77-A:1845-1852.
11. Akiyama H, Kawanabe $\mathrm{K}$, Yamamoto K, et al. Clinical outcomes of cemented double-tapered titanium femoral stems: a minimum 5-year follow-up. J Orthop Sci 2011;16:689-97.

12. Kawate K, Maloney WJ, Bragdon CR, et al. Importance of a thin cement mantle. Autopsy studies of eight hips. Clin Orthop Relat Res 1998;355:70-76.

13. Maloney WJ, Schmalzried T, Harris WH. Analysis of long-term cemented total hip arthroplasty retrievals. Clin Orthop Relat Res 2002;405:70-78.

14. Langlais F, Kerboull M, Sedel L, Ling RS. The 'French paradox'. J Bone Joint Surg [Br]2003;85-B:17-20.

15. EI Masri F, Kerboull L, Kerboull M, Courpied JP, Hamadouche M. Is so-called 'French paradox' a reality?: long-term survival and migration of the Charnley-Kerboull stem cemented line-to-line. J Bone Joint Surg [Br]2010;92-B:342-348.

16. Heiner AD. Structural properties of fourth-generation composite femurs and tibias. J Biomech 2008;41:3282-3284.

17. Hustosky KT, Norman TL, Kish VL, Blaha JD, Gruen TA. The effect of creep on cement hoop stresses and axial displacement of a cemented femoral hip prosthesis in vitro. Trans Orthop Res Soc 1996;21:423.

18. Crowninshield RD, Brand RA, Johnston RC, Milroy JC. The effect of femoral stem cross-sectional geometry on cement stresses in total hip reconstruction. Clin Orthop Relat Res 1980;146:71-77.

19. Zahiri CA, Schmalzried TP, Szuszczewicz ES, Amstutz HC. Assessing activity in joint replacement patients. J Arthroplasty 1998;13:890-895

20. Kwak BM, Lim OK, Kim YY, et al. An investigation of the effect of cement thickness on an implant by finite element stress analysis. Int Orthop 1979;2:315-319.

21. Jasty MJ, Floyd WE, Schiller AL, Goldring SR, Harris WH. Localized osteolysis in stable, non-septic total hip arthroplasty. J Bone Joint Surg [Am] 1986;68-A:912-919.

22. Scheerlinck T, de Mey J, Deklerck R, Noble PC. CT analysis of defect of the cement mantle and alignment of the stem: in vitro comparison of Charnley-Kerboul femoral hip implants inserted line-to-line and undersized in paired femora. J Bone Joint Surg [Br] 2006;88-B:19-25.

23. Janssen D, van Aken J, Scheerlinck T, Verdonschot N. Finite element analysis of the effect of cementing concepts on implant stability and cement fatigue failure. Acta Orthop 2009;80:319-324.

24. Lee C. Properties of bone cement. The mechanical properties of PMMA bone cement. In: Breusch SJ, Malchau H, eds. The Well-Cemented Total Hip Arthroplasty. Theory and Practice. Heidelberg: Springer Medizin Verlag, 1990:85-90.

25. Lewis G. Properties of acrylic bone cement: state of the art review. J Biomed Master Res 1997;38:155-182.

26. Saha S, Pal S. Mechanical properties of bone cement: a review. J Biomed Master Res 1984:18:435-462.

27. Ramos A, Simões JA. The influence of cement mantle thickness and stem geometry on fatigue damage in two different cemented hip femoral prostheses. J Biomech 2009;42:2602-2610

Funding Statement

Funding support: A. Kaneuji and N. Kawahara received a research grant from Zimmer Biomet as chief members of the research department. This study was performed using a research grant that was received by our department from Zimmer Biomet Japan KK.

Author Contribution

E. Takahashi: Performing biomechanical study, Data analysis, Statistical analysis, Manuscript preparation.

A. Kaneuji: Study design, Manuscript preparation, Data analysis, Principal investigator. R. Tsuda: Performing biomechanical study, Statistical analysis, Manuscript preparation.

- Y. Numata : Manuscript preparation, Data analysis.

T. Ichiseki: Manuscript preparation, Statistical analysis.

K. Fukui: Manuscript preparation, Data analysis.

- N. Kawahara: Study design, Manuscript preparation.

ICMJE Conflicts of Interest

None declared

(c) 2017 Kaneuji et al. This is an open-access article distributed under the terms of the Creative Commons Attributions licence (CC-BY-NC), which permits unrestricted use, distribution, and reproduction in any medium, but not for commercial gain, provided the original author and source are credited. 